Agronomía Costarricense 38(1): 11-28. ISSN:0377-9424 / 2014

www.mag.go.cr/rev_agr/index.html www.cia.ucr.ac.cr

\title{
CONCENTRACIÓN FOLIAR DE NUTRIMENTOS EN PLANTACIONES DE TECA EN LA CUENCA DEL CANAL DE PANAMÁ
}

\author{
Rafael Murillo $^{l / *}$, Alfredo Alvarado $^{* *}$, Jean Mark Verjans ${ }^{* * *}$ \\ Palabras clave: Tectona grandis, nutrimentos, concentración, foliar. \\ Keywords: Tectona grandis, nutrients, concentration, foliar.
}

Recibido: 08/05/13

\section{RESUMEN}

Se colectó 89 muestras de hojas en plantaciones de teca en la Cuenca del Canal de Panamá, con el fin de determinar la variación de la concentración foliar de macro y microelementos según el crecimiento de los árboles (bajo, medio y alto crecimiento en volumen.ha-1) y según los grupos de edad de las plantaciones entre 3-4, 5-6 y 7-8 años. Se determinó que el orden de la concentración de los macroelementos en las hojas de teca fue el N (1,9\%), el Ca $(1,1 \%)$, el K $(0,7 \%)$, el $\mathrm{S}(0,2 \%)$ y el $\mathrm{P}(0,1 \%)$ y que el orden de concentración de los microelementos fue el Mn (50), Fe (43), Al (36), B (29), Zn (28), Cu (8) y Mo $(0,4) \mathrm{mg} \cdot \mathrm{kg}^{-1}$, respectivamente. Las concentraciones que se encuentran en niveles claramente bajos son el K, P, Fe, mientras que las de $\mathrm{N}, \mathrm{Ca}, \mathrm{Mg}$ y $\mathrm{Mn}$, pueden considerarse como marginales o ligeramente bajas. Además, los nutrimentos foliares que varían con la edad de los árboles de teca y cuyas variaciones pueden ser explicadas estadísticamente son el $\mathrm{Ca}$, $\mathrm{Mg}, \mathrm{N}, \mathrm{K}, \mathrm{Mn}, \mathrm{Zn}, \mathrm{Fe}$ y Al. Las concentraciones foliares de $\mathrm{Ca}, \mathrm{Mg}, \mathrm{Mn}, \mathrm{Fe}$ y $\mathrm{Al}$ muestran una tendencia a aumentar con la edad, mientras que las de $\mathrm{N}, \mathrm{K}$ y $\mathrm{Zn}$ tienden a disminuir. El contenido foliar de $\mathrm{Ca}$ y $\mathrm{Mn}$ en las categorías de crecimiento alto y bajo de la teca es diferente estadísticamente $(\mathrm{p}<0,01)$, con valores

\footnotetext{
1 Autor para correspondencia. Correo electrónico: rafael.murillo.cruz@una.cr Universidad Nacional de Costa Rica. Heredia, Costa Rica.
}

Aceptado: 24/09/13

\begin{abstract}
Foliar nutrient concentration in teak plantations at the Panama Canal watershed. Foliar concentration of macro- and micronutrients in teak plantations at the Panama Canal Watershed was measured on sites classed as high, medium, and low in growth (volume.ha-1 ${ }^{-1}$. For the same purpose, plantations where grouped by age as young (3-4 years), medium (5-6 years) and late (7-8 years). Foliar macro-nutrients concentration followed the order $\mathrm{N}(1,9 \%), \mathrm{Ca}(1.1 \%), \mathrm{K}(0.7 \%)$, $\mathrm{S}(0.2 \%)$ and $\mathrm{P}(0.1 \%)$, while micro-nutrients concentrations decreased in the order Mn (50), $\mathrm{Fe}$ (43), Al (36), B (29), Zn (28), $\mathrm{Cu}$ (8), and Mo (0.4) mg.kg-1. The K, P and Fe concentrations were clearly identified as low, while $\mathrm{N}, \mathrm{Ca}, \mathrm{Mg}$ and $\mathrm{Mn}$ values can be considered as marginal to slightly low. The Ca, Mg, N, K, Mn, Zn, Fe and Al concentrations varied statistically with plantation age in different ways; foliar concentrations of $\mathrm{Ca}$, $\mathrm{Mg}, \mathrm{Mn}, \mathrm{Fe}$ and $\mathrm{Al}$ tend to increase with age, while those of $\mathrm{N}, \mathrm{K}$ and $\mathrm{Zn}$ tend to diminish with age. Concentrations of $\mathrm{Ca}$ and $\mathrm{Mn}$ in the leaves of trees of sites with high and low growth differed statistically $(\mathrm{p}<0.01)$, with Ca values of $1.18 \%$ and $0.99 \%$, in the high and low growth categories, respectively, and with $\mathrm{Mn}$ values of 41 and 65 mg.kg ${ }^{-1}$ in sites of high and low growth rates, respectively.
\end{abstract}

\footnotetext{
** Universidad de Costa Rica. San José, Costa Rica.

*** Ecoforest Panamá
} 
para el Ca de $1,18 \%$ y $0,99 \%$ en categoría alta y baja, respectivamente, y para el Mn de 41 y 65 mg.kg ${ }^{-1}$ en categoría de crecimiento alta y baja, respectivamente.

\section{INTRODUCCIÓN}

Los valores de concentración foliar de teca para edades menores de 3,5 años en Panamá (Mollinedo et ál. 2005) son más bajos que los mencionados para plantaciones de esta especie en África (Nwoboshi 1984, Drechsel y Zech 1991), pero similares a las concentraciones foliares encontradas en plantaciones de teca en Costa Rica (Montero y Kanninen 2006).

Drechsel y Zech (1991) tomaron en cuenta las variaciones foliares encontradas en África y desarrollaron criterios de suficiencia, marginalidad y exceso de nutrimentos, pero no mencionan la variación de la concentración con la edad de la plantación, como si lo hacen Montero y Kanninen (2006) y Nwoboshi (1984), quienes encontraron que las concentraciones de $\mathrm{K}$ y $\mathrm{N}$ son mayores en los árboles de 1 año de edad que en edades mayores. Otros autores, aportan un poco más de conocimiento sobre la variación de la concentración foliar de nutrimentos en otros países (Zech y Kaupenjohann 1990, Koppad y Rao 2003).

Vásquez y Ugalde (1995) encontraron en plantaciones de teca en el Pacífico estacionalmente seco de Costa Rica que la concentración foliar de nutrimentos es mayor en suelos con alto contenido de bases, como los Vertisoles bien drenados, que en los Ultisoles rojos y ácidos. Así, los elementos de mayor disponibilidad como el $\mathrm{Ca}$ en los suelos vérticos serán los de mayor concentración a nivel foliar, siempre y cuando los suelos no presenten un contenido de arcilla expandible superior al 55\% que altere sus mecanismos de absorción.

La concentración de nutrimentos en la hoja varía según su posición en la copa, en las ramas y la época de crecimiento (Sampaio y Huber 1999, Evans 1979). Evans (1979) encontró en árboles de Gmelina arborea que el N, P, Zn y B decrecen desde la parte más externa de la copa hasta la más interna, es decir con el aumento en la sombra, mientras que el Ca y el Fe crecieron.

La aplicación de fertilizantes al suelo causó una diferencia de concentración significativa del $\mathrm{N}$ y el $\mathrm{K}$ foliar en árboles de teca de 2 años, la cual aumentó desde 1,60 hasta 2,58\% y de 0,93 hasta $1,38 \%$, respectivamente (Koppad y Rao 2003).

La concentración foliar de nutrimentos, permite relacionar las variables de crecimiento con la nutrición de las plantaciones. El contenido de $\mathrm{K}$ foliar explicó el $57 \%$ de la variación del área basal de la teca en suelos moderada y pobremente drenados en Venezuela (Hernández et ál. 1993), mientras que en Guatemala el K correlacionó en un $76 \%$ con el incremento medio anual en diámetro (Rodas 2006). El K y el P foliar presentaron valores de concentración mayores en suelos de drenaje moderado $(2,25 \%$ y $0,35 \%$ respectivamente) que en suelos de drenaje pobre (Hernández et ál. 1993). Negi et ál. (1990), encontraron valores de $0,83 \%$ de $\mathrm{K}$ foliar en hojas de árboles de teca con crecimiento a una tasa de $7,9 \mathrm{~m}^{3} \mathrm{ha}^{-1}$. año ${ }^{-1}$, cifras más elevadas que las encontradas en India y Benín (Vimal et ál. 2003, Zech y Kaupenjohann 1990). La teca que creció en suelos rojos ácidos en Benín, mostró un crecimiento promedio de $9,3 \mathrm{~m}^{3} \mathrm{ha}^{-1}$.año ${ }^{-1}$ asociado a contenidos foliares deficientes de K $(0,29 \%)$, medios de $\mathrm{P}$ y Ca $(0,11 \%$ y $1,09 \%)$ y medios a altos de $\mathrm{Mg}$ (0,42\%) (Zech y Kaupenjohann 1990).

En la India, Vimal et ál. (2003) no encontraron relaciones satisfactorias entre los contenidos foliares de nutrimentos y el crecimiento de la teca de menos de 20 años; los autores desarrollaron un modelo lineal con el $\mathrm{N}$ (coeficiente negativo) para explicar el desarrollo de la teca $\mathrm{y}$ 
lo atribuyeron al efecto de dilución de la concentración de $\mathrm{N}$ en las hojas al incrementarse el crecimiento. En este mismo estudio, se encontró una relación $\left(R^{2}=0,41\right)$ entre el incremento corriente anual del área basal y los nutrimentos $\mathrm{Zn}, \mathrm{Fe}, \mathrm{N}$ y $\mathrm{Mg}$ en las hojas, todos con coeficientes negativos.

La concentración de los microelementos en las hojas de teca ha sido poco estudiada, aunque se ha demostrado su importancia debido a que la deficiencia de dichos micronutrimentos produce un retardo en el crecimiento de la planta, reducción en el número de hojas saludables, acortamiento entre nudos, hojas anormalmente más grandes y disminución del crecimiento apical y de la raíz. La mayor reducción del crecimiento se debió a deficiencias de Mo, mientras que la mayor disminución de hojas saludables se atribuyó a deficiencias de Fe y la mayor disminución en el crecimiento de la raíz a deficiencias de $\mathrm{Cu}$ (Sujatha 2003).

El crecimiento de los árboles de teca puede ser inhibido por concentraciones altas de los elementos $\mathrm{Fe}, \mathrm{Al}$ y parcialmente el $\mathrm{Mn}$ (Drechsel y Zech 1992); sin embargo el Al foliar es un mal indicador de la toxicidad por Al (Dreschel y Zech 1994). La toxicidad por Al inhibe el crecimiento radicular y puede inducir deficiencia de B (Lenoble et ál. 1996a), además de reducir la eficiencia del $\mathrm{P}, \mathrm{K}, \mathrm{Ca}$ y $\mathrm{Mg}$ (Lenoble et ál. 1996b), Vitorello et ál. 2005. Como respuesta de las hojas a la toxicidad por Al los arboles sufren modificaciones estructurales y celulares, reducción de la apertura de los estomas, disminución de la actividad fotosintética, clorosis, necrosis y reducción de la biomasa de las plantas (Vitorello et ál. 2005).

El objetivo del presente trabajo fue determinar y clasificar los valores de concentración foliar de macroelementos (N, P, K, Ca, Mg, S) y microelementos ( $\mathrm{Fe}, \mathrm{Cu}, \mathrm{Zn}, \mathrm{B}, \mathrm{Al}, \mathrm{Mn}, \mathrm{Mo}$ ) en la teca de 36 a 96 meses de edad.

\section{MATERIALES Y MÉTODOS}

Las muestras foliares utilizadas en el presente trabajo provienen de plantaciones en la cuenca del Canal de Panamá, entre las coordenadas geográficas $9^{\circ} 00^{\prime}$ y $9^{\circ} 10^{\prime}$ norte, $79^{\circ} 45^{\prime}$ y $80^{\circ} 00^{\prime}$ oeste. Los suelos son Ultisoles mayormente rojos y ácidos en la zona de vida bosque húmedo tropical según la clasificación de Holdridge, la temperatura promedio anual es de $26,3^{\circ} \mathrm{C}$ y la precipitación promedio anual es de $2880 \mathrm{~mm}$ (TLBG 2006). El sitio de muestreo consistió en una red de parcelas permanentes preestablecidas y clasificadas según el incremento medio anual en volumen, como bajo $\left(2,9-5,3 \mathrm{~m}^{3} \mathrm{ha}^{-1} \cdot \mathrm{anno}^{-1}\right)$, medio $\left(5,4-9,3 \mathrm{~m}^{3} \mathrm{ha}^{-1} \cdot\right.$ año-1 $)$ y alto $\left(9,4-13,3 \mathrm{~m}^{3}\right.$ $\left.\mathrm{ha}^{-1} \cdot \mathrm{año}^{-1}\right)$. Se seleccionaron 30 parcelas en sitios de crecimiento bajo, 30 parcelas en sitios de crecimiento medio y 29 en sitios de crecimiento alto, para un total de 89 parcelas, distribuidas equitativamente en cada ámbito de años (3-4, 5-6 y 7-8). Las anteriores categorías de crecimiento corresponden a crecimientos muy bajos, bajos y medios a nivel Centroamericano y el Caribe, según información reportada por otros autores (FAO 1977, Keogh 1979, Vásquez y Ugalde 1995, Vallejos 1996, Montero 1999, Ávila 2003, Vaides 2004). Alrededor de cada una de las parcelas se realizó un muestreo de hojas de teca en 4 árboles dominantes (5 hojas por árbol) localizados a no más de $15 \mathrm{~m}$ del borde de cada parcela permanente seleccionada. Cada muestra se compuso de 20 hojas obtenidas del tercio superior completamente iluminado del árbol y a partir del tercer par de hojas desde el ápice hacia el centro de la rama.

En el campo, las muestras se almacenaron en bolsas de papel debidamente identificadas, se guardaron en hieleras para evitar el deterioro y se enviaron al laboratorio del Centro de Investigaciones Agronómicas de la Universidad de Costa Rica (CIA). El análisis de las muestras foliares se realizó por medio de la metodología de digestión húmeda por espectrofotometría de emisión atómica con plasma para el $\mathrm{Ca}, \mathrm{Mg}, \mathrm{K}, \mathrm{P}, \mathrm{S}, \mathrm{B}, \mathrm{Al}$, $\mathrm{Zn}, \mathrm{Cu}, \mathrm{Mn}, \mathrm{Mo}$ y Fe, el N por el método Microkjeldahl (Henríquez et ál. 1995).

Se realizó las curvas de regresión entre la concentración foliar, la clase de edad y la clase de sitio para cada uno de los elementos y se hizo la prueba de comparación de medias para cada uno 
de los mismos, según el crecimiento en volumen sin distinción de la edad y según la edad sin distinción del crecimiento en volumen. Para cada uno de los elementos, se llevó a cabo un análisis para cuantificar su suficiencia según estándares internacionales (Dreschel y Zech 1991) sin considerar la edad o el nivel productivo de los sitios.

Con toda la población de datos, se realizó la distribución normal de frecuencias, de manera que se pudiera calcular la normalidad estadística de los valores y definir ámbitos como moderadamente bajos (valores que representan el $10 \%$ del área a la izquierda de la curva normal), adecuados (el 80\% de los valores del área de la curva) y moderadamente altos (valores que representan el $10 \%$ del área a la derecha de la curva normal) de la concentración de cada uno de los elementos. Debido a que no se encontró síntomas de deficiencia foliar en las plantaciones, esta separación asume que la concentración de todos los elementos comparados fue de suficiencia, lo cual puede o no ser así, según los criterios de suficiencia definidos por Dreschel y Zech (1991). Los datos se analizaron con el programa: Estadística StatSoft, Inc. (2001) STATISTICA (Data Analysis Software System), versión 6.

\section{RESULTADOS Y DISCUSIÓN}

\section{Variación de la concentración foliar de nutrimentos}

En el Cuadro 1, se presentan los valores mínimo, promedio y máximo y el coeficiente de variación de las concentraciones de todos los elementos analizados a nivel foliar en las plantaciones estudiadas, indiferentemente de la edad de la plantación o de la calidad del sitio. La mayoría de los valores presenta coeficientes de variación que podrían considerarse medios a altos, según el elemento y el criterio establecido para la mayoría de cultivos agrícolas por el Comité de Laboratorios de Costa Rica (Bertsch et ál. 2005).

Cuadro 1. Estadísticos para el contenido foliar de nutrimentos en plantaciones de teca de 3 a 8 años en la Cuenca del Canal de Panamá.

\begin{tabular}{|c|c|c|c|c|c|c|}
\hline \multicolumn{2}{|c|}{ Elemento } & $\mathrm{n}$ & Media & $\mathrm{CV}(\%)$ & Mín & Máx \\
\hline \multirow{7}{*}{$\begin{array}{c}\text { Macro- } \\
\text { elementos } \\
(\%)\end{array}$} & $\mathrm{N}$ & 89 & 1,9 & 13 & 1,36 & 2,61 \\
\hline & $\mathrm{Ca}$ & 89 & 1,06 & 25 & 0,50 & 1,84 \\
\hline & K & 89 & 0,66 & 25 & 0,26 & 1,02 \\
\hline & $\mathrm{Mg}$ & 89 & 0,27 & 37 & 0,12 & 0,57 \\
\hline & $\mathrm{S}$ & 89 & 0,20 & 11 & 0,14 & 0,25 \\
\hline & $\mathrm{P}$ & 89 & 0,12 & 12 & 0,09 & 0,16 \\
\hline & $\mathrm{Na}$ & 89 & 0,01 & 33 & 0,003 & 0,01 \\
\hline \multirow{7}{*}{$\begin{array}{l}\text { Micro- } \\
\text { elementos } \\
\left(\mathrm{mg} \cdot \mathrm{kg}^{-1}\right)\end{array}$} & $\mathrm{Mn}$ & 89 & 50 & 48 & 21 & 135 \\
\hline & $\mathrm{Fe}$ & 89 & 43 & 14 & 33 & 72 \\
\hline & $\mathrm{Al}$ & 89 & 36 & 28 & 20 & 94 \\
\hline & B & 89 & 29 & 21 & 16 & 54 \\
\hline & $\mathrm{Zn}$ & 89 & 28 & 31 & 16 & 67 \\
\hline & $\mathrm{Cu}$ & 89 & 8 & 24 & 4 & 13 \\
\hline & Mo & 89 & 0,35 & 24 & 0,2 & 0,64 \\
\hline
\end{tabular}

$\mathrm{DE}=$ desviación estándar, $\mathrm{EE}=$ error estándar, $\mathrm{CV}=$ coeficiente de variación, Mín=valor mínimo, Máx=valor máximo, Media, DE, EE, Mín, y Máx están en unidades de cada elemento. 
Al comparar los valores de concentración foliar de los diferentes elementos analizados con los parámetros de suficiencia foliar desarrollados por Dreschel y Zech (1991) para plantaciones de teca en África, se considera que las concentraciones de N, Ca, Zn, B y S son adecuadas (Figura 1) y la concentración de $\mathrm{Mg}$ es ligeramente superior al nivel crítico (Figura 2), mientras que las concentraciones de $\mathrm{K}, \mathrm{P}, \mathrm{Fe}$ y $\mathrm{Cu}$ son claramente bajas (Figura 3). Los valores de Na y Mo no se compararon con los datos de Dreschel y Zech (1991) quienes no los consideran en su estudio y
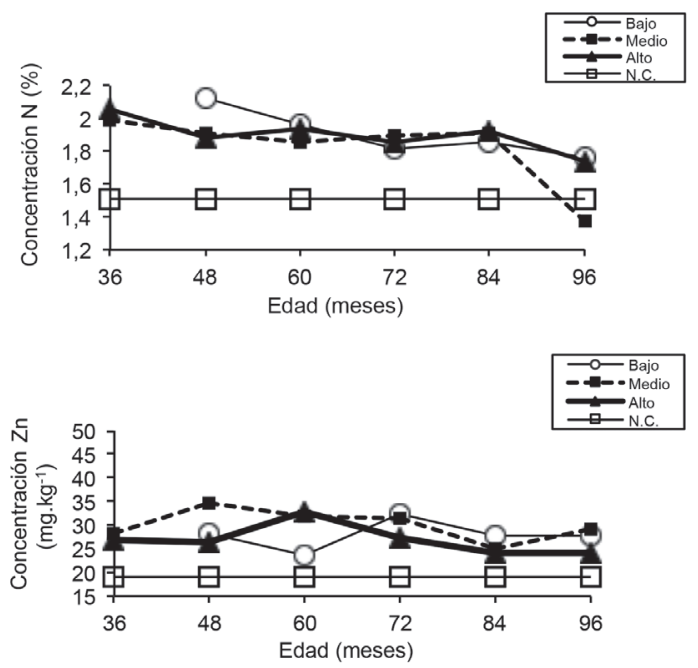

por tanto representan un nuevo aporte a la literatura. Las concentraciones de Al están por debajo del rango de exceso (Figura 4), sin embargo Dreschel y Zech (1994) mencionan que el Al foliar no es buen indicador de toxicidad por Al, razón por la cual se tomó este dato con reservas. Las concentraciones de Mn aumentan conforme disminuye la calidad de sitio (volumen total $\mathrm{m}^{3} \cdot \mathrm{ha}^{-1}$ ) (Figura 4) y aquellas de los sitios de bajo crecimiento se encuentran en concentraciones mayores que el nivel de suficiencia crítico determinado por Dreschel y Zech (1991).
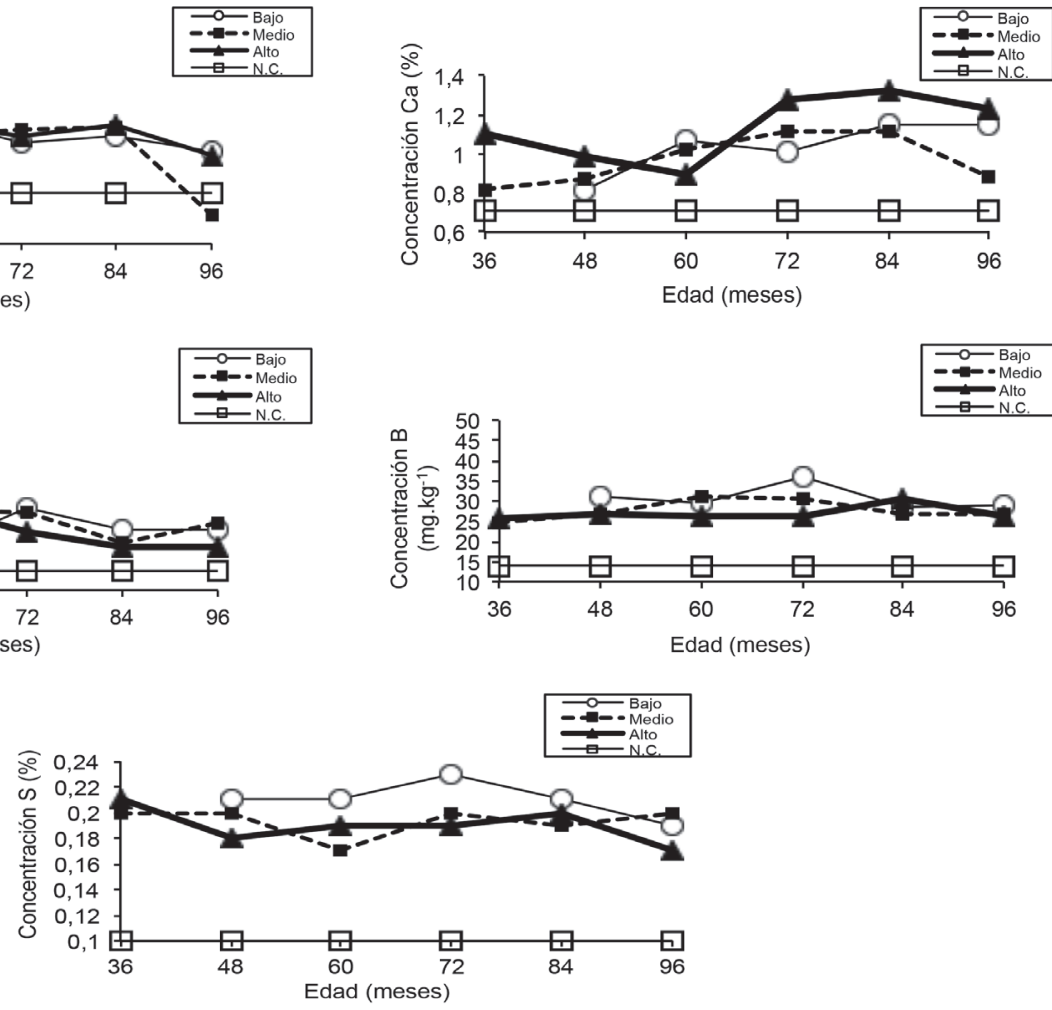

Fig. 1. Curvas de concentración foliar de N, Ca, Zn, B y S en plantaciones de teca según la edad y la calidad de sitio en la Cuenca del Canal de Panamá.

N.C.= Nivel crítico según Drechsel y Zech (1991). 


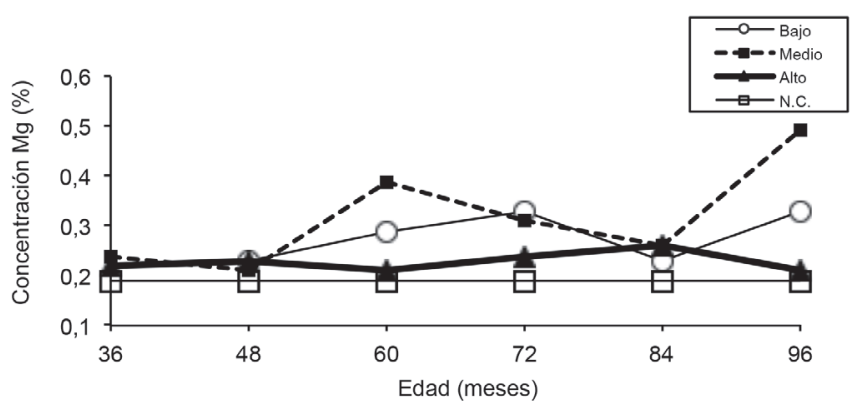

Fig. 2. Curva de concentración foliar del Mg en plantaciones de teca según la edad y la calida de sitio de la Cuenca del Canal de Panamá.

NC= Nivel crítico según Drechsel y Zech (1991).
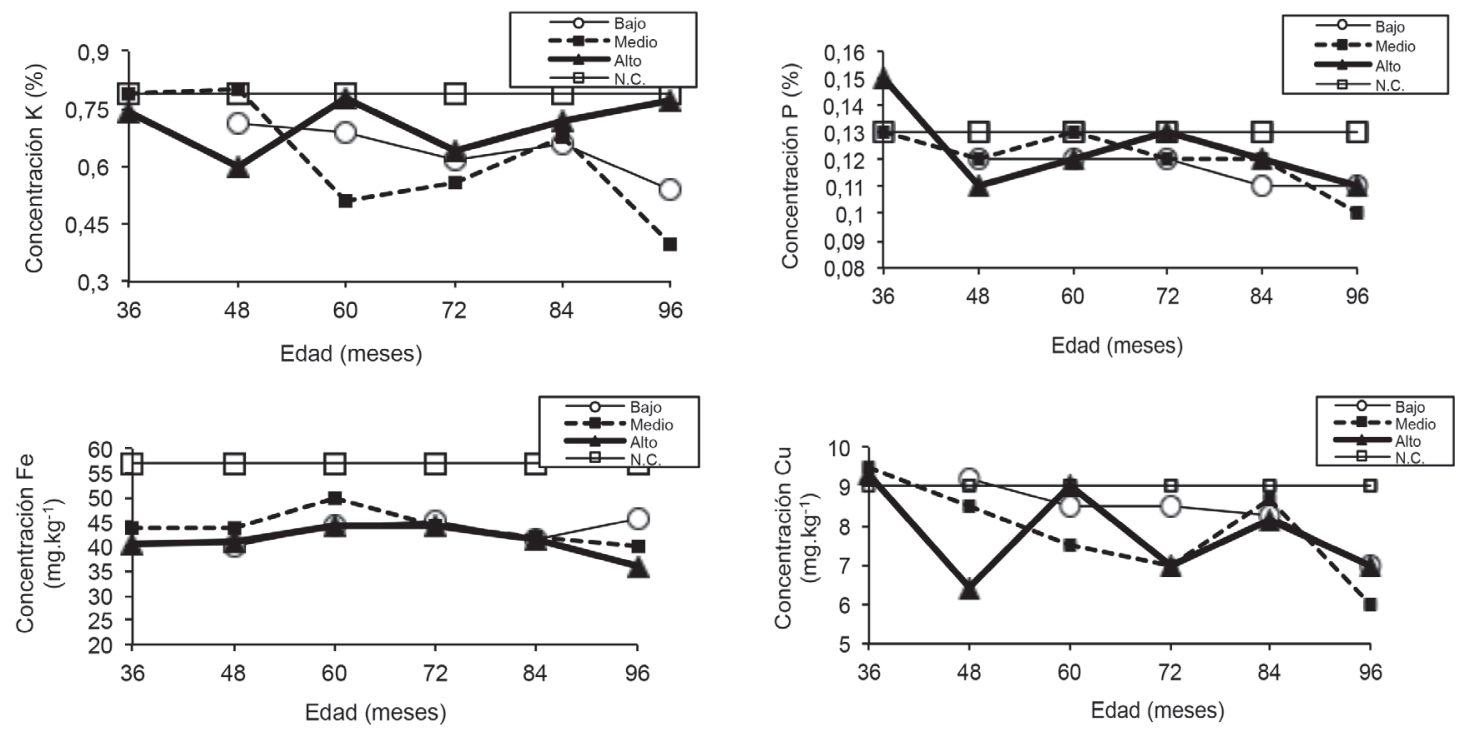

Fig. 3. Curvas de concentración foliar de K, P, Fe y Cu en plantaciones de teca según la edad y la calidad de sitio en la Cuenca del Canal de Panamá. N.C.= Nivel crítico
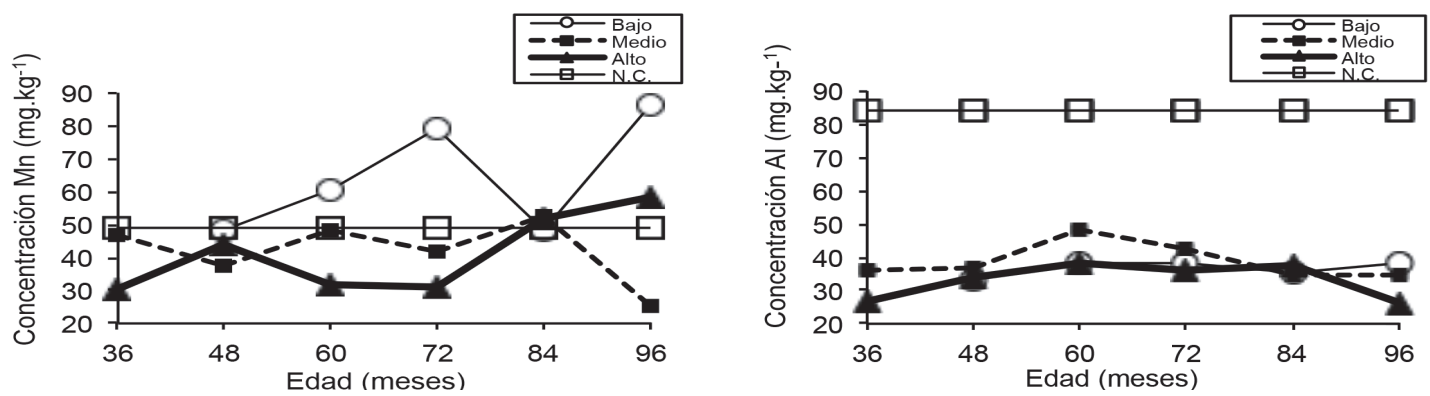

Fig. 4. Curvas de concentración foliar de Al y Mn en plantaciones de teca según la edad y la calidad de sitio en la Cuenca del Canal de Panamá. 
Al realizar la distribución de frecuencia de los datos por elemento, se encontró que los elementos analizados presentan una distribución normal, excepto el $\mathrm{Zn}$ y el Mn. En términos de concentración foliar el $79 \%$ de las muestras de $\mathrm{K}$ y el $92 \%$ de las muestras de P (Figuras 5 y 6 ) están por debajo del nivel crítico mencionado por Dreschel y Zech (1994).

Frecuencia absoluta de la concentración foliar de K

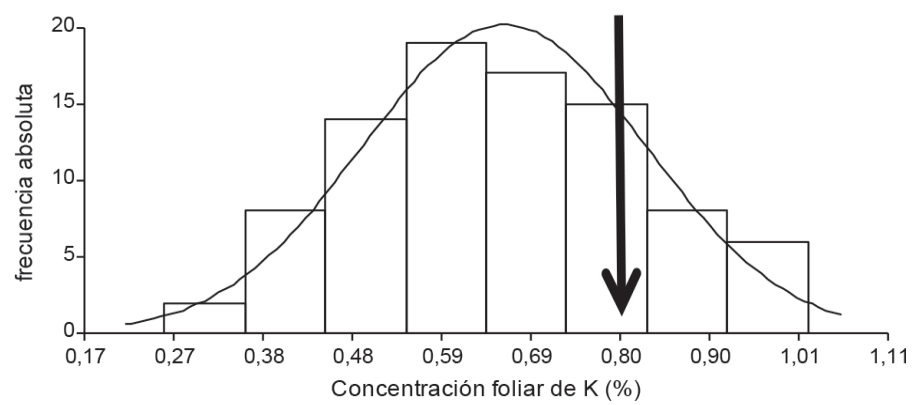

Fig. 5. Distribución de frecuencia de todas las muestras de concentración foliar de K y el nivel crítico según Dreschel y Zech (1991).

La flecha indica el nivel crítico.

Distribución de la frecuencia absoluta de la concentración foliar de P

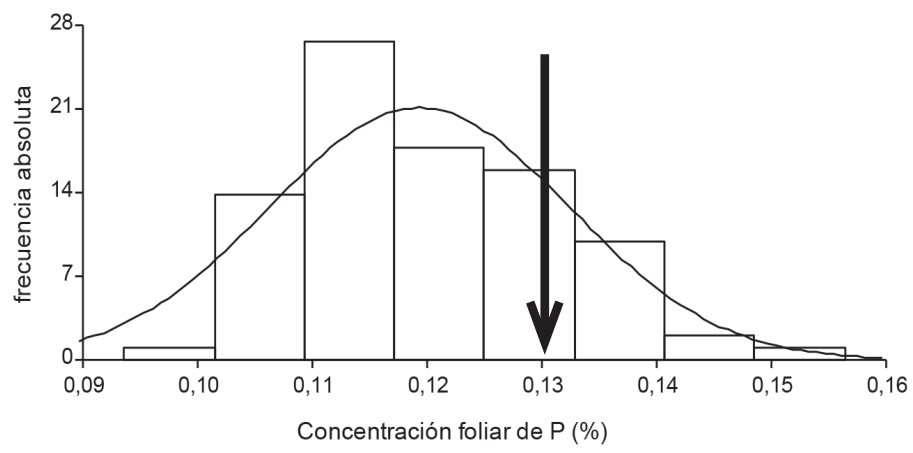

Fig. 6. Distribución de frecuencia de todas las muestras de concentración foliar de P y el nivel crítico según Dreschel y Zech (1991).

La flecha indica el nivel crítico.

\section{Efecto de la calidad de sitio sobre la concentración foliar de nutrimentos de la teca}

Al agrupar los datos por calidad de sitio (baja, media, alta) (Cuadros 2 y 3), se encuentra que las diferencias de concentración en las calidades de sitio no son significativas $(\mathrm{p}>0,05)$ para varios de los elementos estudiados, excepto para el $\mathrm{S}, \mathrm{Ca}, \mathrm{Mn}, \mathrm{B}$ y $\mathrm{Cu}(\mathrm{p}<0,05)$. En los sitios con crecimiento bajo de la teca, los valores de concentración foliar de Ca son menores $(0,99 \%)$ que los valores de concentración en los sitios de crecimiento alto (1,18\%) (Cuadro 2), es decir a mayor concentración de $\mathrm{Ca}$ en las hojas mayor es el crecimiento de los árboles de teca. Según Alvarado y Fallas (2004) la teca es una especie que necesita de alta saturación de calcio en el suelo (valores de 68\%) para desarrollarse bien. Los valores de concentración foliar del Mn en los sitios de crecimiento alto tienen la tendencia a estar por debajo de los valores de concentración del Mn en los sitios de crecimiento bajo. 


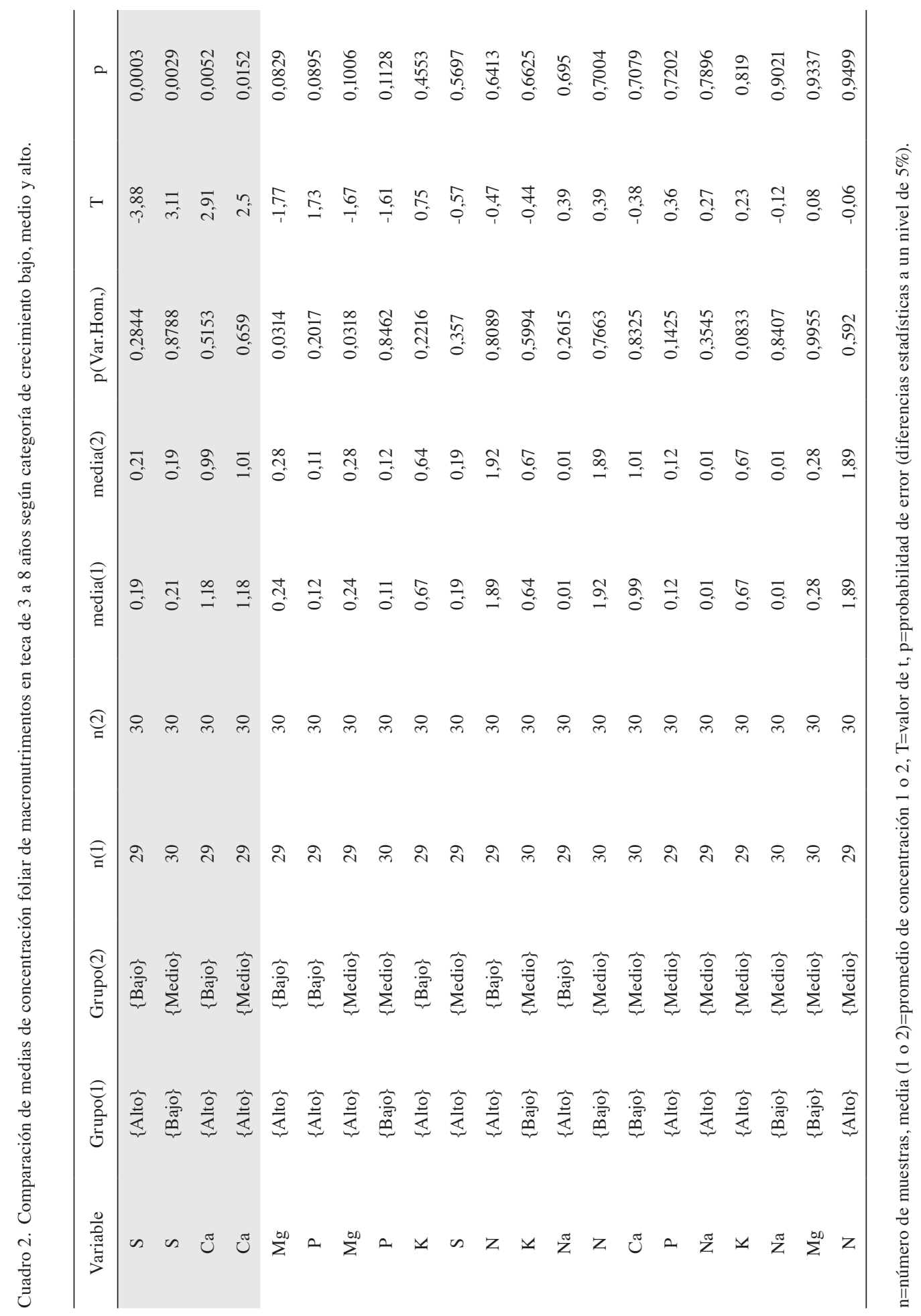




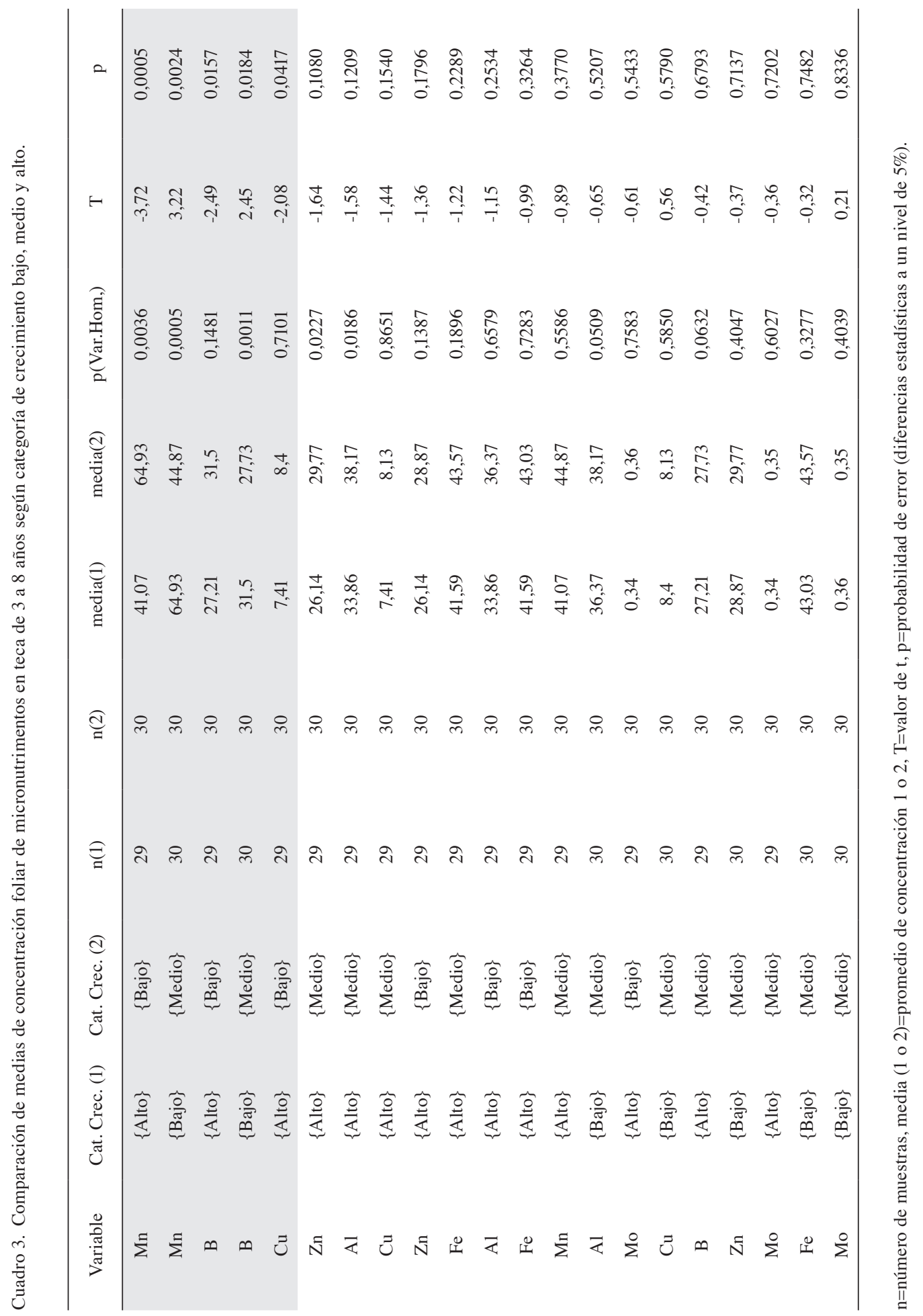


La concentración foliar de Mn en los sitios de bajo crecimiento (65 mg. $\left.\mathrm{kg}^{-1}\right)$ es estadísticamente diferente $(p<0,01)$ de la concentración en los sitios de alto crecimiento (41 mg.kg-1). Para los otros microelementos las diferencias no son estadísticamente significativas $(\mathrm{p}>0,05)$.

\section{Efecto de la edad de la plantación sobre la concentración foliar de nutrimentos de la teca}

El comportamiento de las curvas de concentración foliar y su relación con la edad de la plantación es variable. El $\mathrm{Ca}\left(\mathrm{R}^{2}=0,30\right)$ y el $\mathrm{Mg}$ $\left(\mathrm{R}^{2}=0,17\right)$ tienen la tendencia a aumentar con la edad de los árboles, mientras que el $\mathrm{N}\left(\mathrm{R}^{2}=0,49\right)$, $\mathrm{P}\left(\mathrm{R}^{2}=0,43\right)$ y $\mathrm{K}\left(\mathrm{R}^{2}=0,20\right)$ disminuyen con la edad, sin embargo, los valores de $\mathrm{R}^{2}$ para todos los casos son bajos. La variación en la concentración foliar según la edad es explicada en parte por la dispersión de los datos $(\mathrm{CV})$ según la concentración foliar por clase de crecimiento. El incremento del Ca y Mg foliar con la edad ya había sido reportado para plantaciones de teca en Costa Rica por Montero y Kanninen (2006), mientras que la disminución de $\mathrm{N}, \mathrm{P}$ y $\mathrm{K}$ ha sido también documentada durante el primer año de crecimiento (Nwoboshi 1984), durante los primeros 4-6 años (Drechsel y Zech 1994) y en árboles de 2-8 años vs 9-45 años (Montero y Kanninen 2006).

Las mayores concentraciones de P se observan en las edades más jóvenes, tanto en sitios de alto como en algunos de bajo crecimiento. Esta información tiene relevancia si se desea estimar la exportación del fósforo del sistema al realizar raleos a muy temprana edad, o también, con la tendencia actual a cosechar la teca cada vez más temprano.

La concentración del $\mathrm{N}$ tiene la tendencia a disminuir con la edad y una ligera tendencia a disminuir con el aumento en la clase de crecimiento (Figura 1), sin embargo, la mayor concentración ocurre en edades más jóvenes y el efecto en la concentración foliar provocado por la edad es mayor que el efecto provocado por la categoría de crecimiento.

El Ca es el macronutrimento que presenta la tendencia más clara respecto de la edad y la clase de crecimiento. Conforme aumenta la edad y el crecimiento, mayor es la concentración de $\mathrm{Ca}$ en las hojas. Las concentraciones más bajas se observan en la categoría de bajo crecimiento y en edades jóvenes. El análisis de la concentración foliar de nutrimentos según los grupos de edad (Cuadro 4) muestra que la variabilidad del $\mathrm{Ca}$ entre el grupo de árboles de 3 y 4 años es estadísticamente diferente $(\mathrm{p}<0,01)$ del grupo de edad de 5 y 6 años y del grupo de edad de 7 y 8 años. El N, Mg (Cuadro 4) y Mn (Cuadro 5) registraron también diferencias significativas $(\mathrm{p}<0,05)$ entre los grupos de edad 3-4 y 7-8 años. El análisis de la concentración del Ca y $\mathrm{N}$ en el grupo de edad de 3-4 años, muestra que la concentración de $\mathrm{Ca}$ es mayor en sitios de crecimiento alto y la concentración de $\mathrm{N}$ es mayor en sitios de crecimiento bajo (Figura 7). 


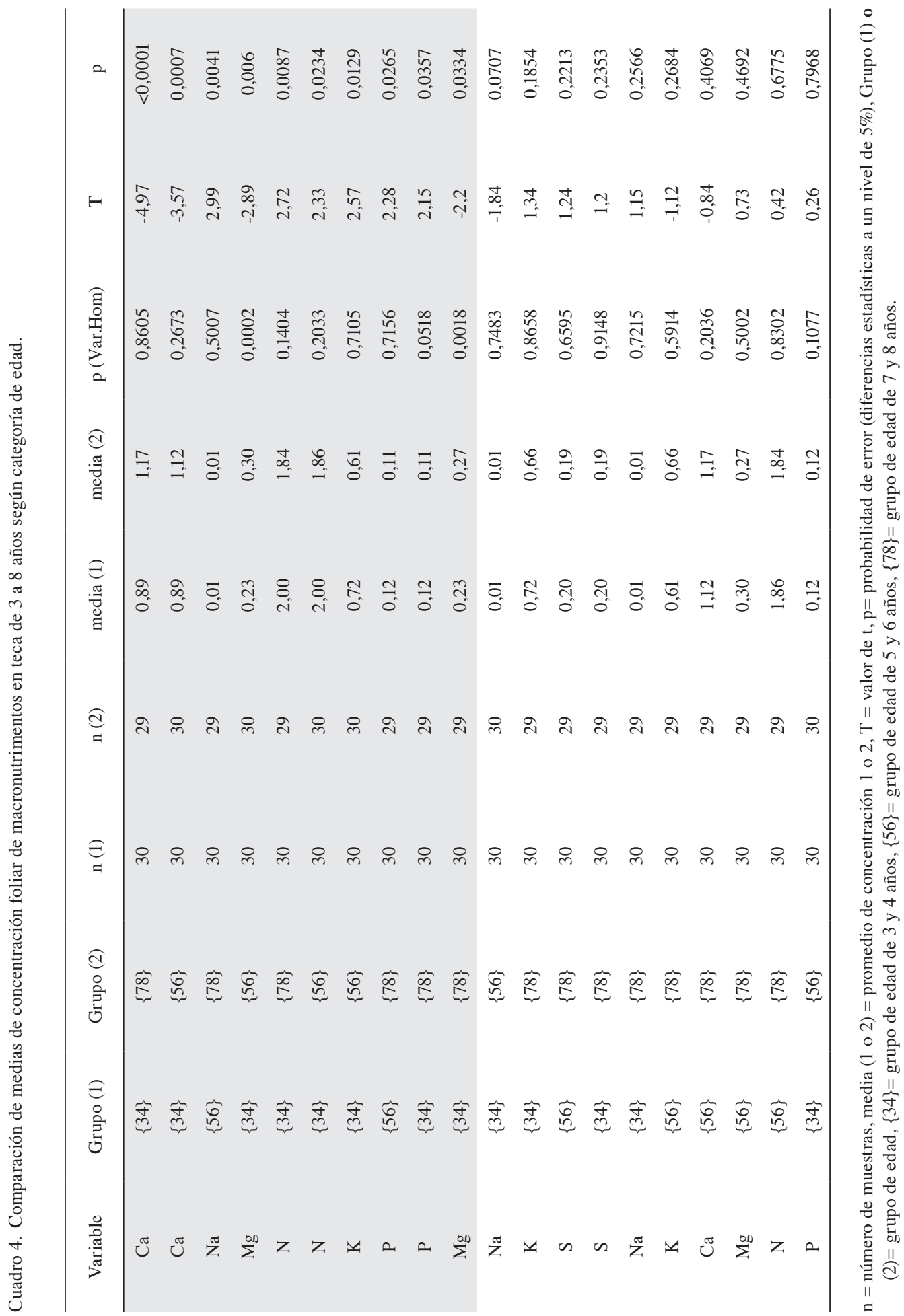

Agronomía Costarricense 38(1): 11-28. ISSN:0377-9424 / 2014 


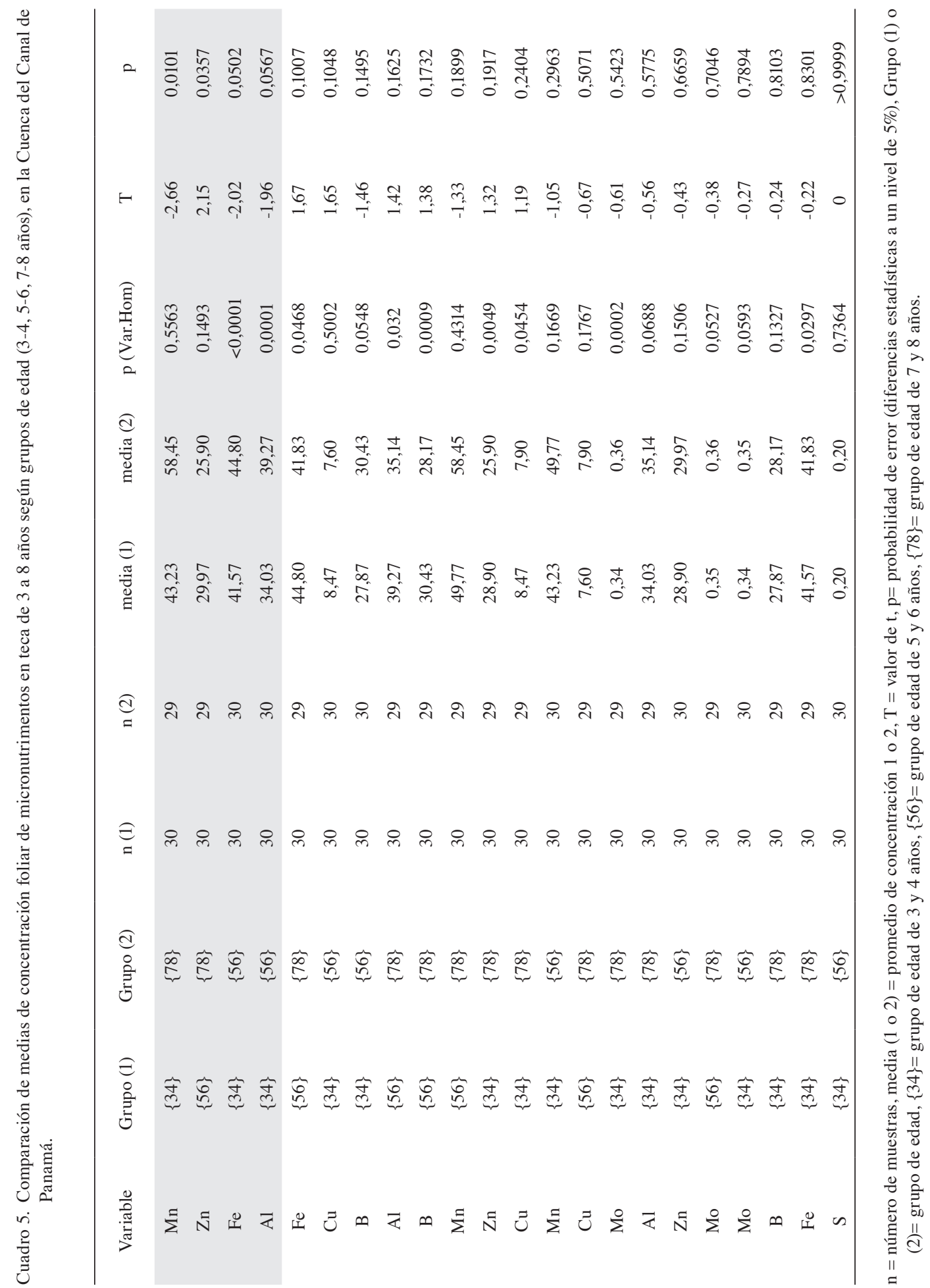



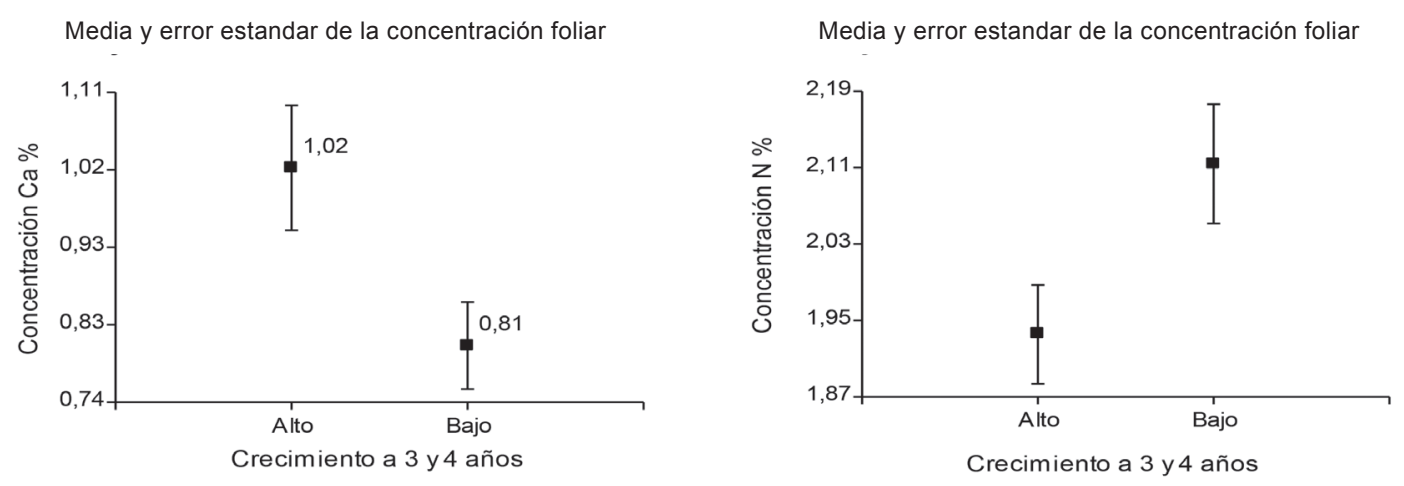

Fig. 7. Promedio y error estándar de las concentraciones de Ca y $\mathrm{N}$ foliar en sitios de alta y baja tasa de crecimiento a la edad de 3 y 4 años.

En relación con la variación de los microelementos en el componente foliar de la teca, se ha mencionado que el $\mathrm{Cu}$ y el $\mathrm{Zn}$ permanecen relativamente estables con la edad (Drechsel y Zech 1994) y que el $\mathrm{Cu}$ tiende a disminuir en plantaciones de Costa Rica (Montero y Kanninen 2006).

Como puede observarse en el Cuadro 5, el Zn tiende a disminuir después del año $5(\mathrm{p}=0,05)$ en los grupos de edad de 5-6 años y 7-8 años (30 a $26 \mathrm{mg} \cdot \mathrm{kg}^{-1}$ ), sin embargo en términos absolutos la diferencia es pequeña, valores considerados normales para otras especies arbóreas. Se ha reportado en la literatura que la concentración de $\mathrm{Zn}$ en árboles de hoja ancha oscila comúnmente entre 15 y 50 mg. $\mathrm{kg}^{-1}$ e inusualmente entre 65 y 200 mg. $\mathrm{kg}^{-1}$ en árboles que crecen en suelos ácidos en bosques húmedos (Boardman y Mcguire 1990). La concentración foliar de Zn en coníferas se ha reportado que oscila entre 15 y 65 mg.kg-1 (Raupach 1975 citado por Boardman y Mcguire 1990).

Con base en los datos foliares de $\mathrm{P}, \mathrm{N}$, $\mathrm{Ca}$ y $\mathrm{Al}$ en las plantaciones de teca estudiadas se elaboró la curva de mejor ajuste para cada uno de estos elementos (Figura 8), que muestran además, el nivel crítico definido por Dreschel y Zech (1991). La variación en la concentración foliar del Ca, N, P y Al podría explicarse como una consecuencia de la edad de la plantación en un $51 \%, 49 \%, 44 \%$ y $38 \%$ respectivamente. Las curvas de mejor ajuste para el $\mathrm{P}$ y el $\mathrm{K}$ foliar de las plantaciones de teca de Costa Rica (Montero y Kanninen 2006) no se ajustan a los datos de Panamá probablemente debido a que los contenidos de P y K foliar en Panamá son menores a los contenidos foliares de teca en Costa Rica. 

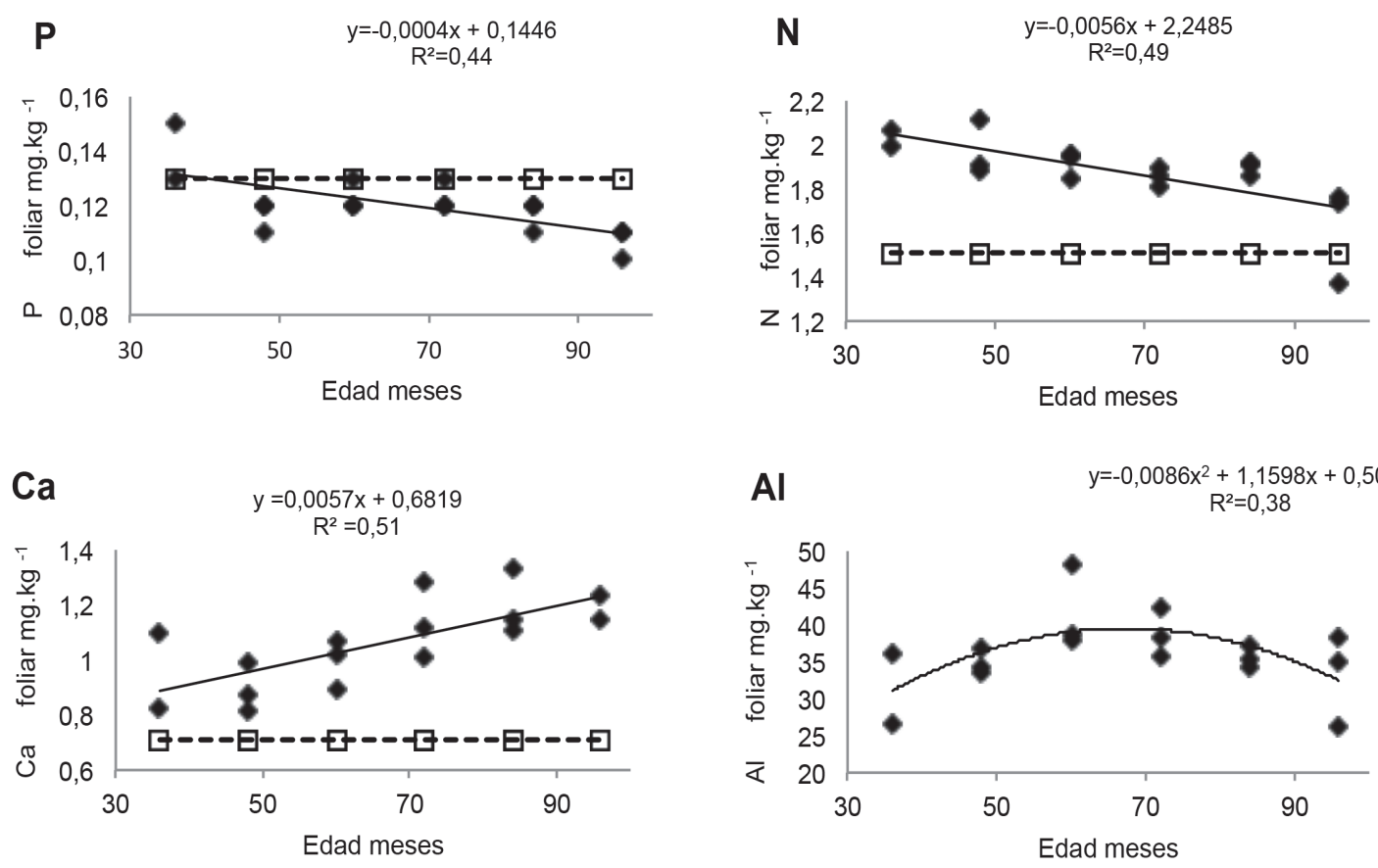

Nivel crítico.

Fig. 8. Tendencia de la concentración foliar del P, N, Ca y Al en teca de 36 a 96 meses en la Cuenca del Canal de Panamá.

\section{Cantidad de nutrimentos foliares en la teca}

Los macroelementos que se registraron en mayor concentración foliar en este estudio fueron $\mathrm{N}$, Ca y $\mathrm{K}$ y en menor cantidad $\mathrm{Mg}, \mathrm{P}$ y $\mathrm{S}$ (Figura 9). Similares resultados han sido reportados en la India y Nigeria (Nwoboshi 1983, 1984, Negi et ál. 1990, Dreschel y Zech 1991) y en Costa Rica por Montero y Kanninen (2006). La concentración foliar de nutrimentos

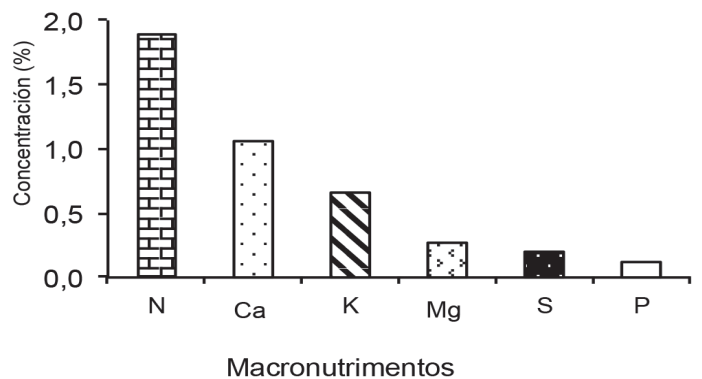

para teca varía más en suelos fértiles de la India (Negi et ál. 1990) y en Nigeria (Nwoboshi 1983, 1984) que en los suelos ácidos encontrados en el presente trabajo en la cuenca del Canal de Panamá. Las variaciones en concentración foliar encontradas, posiblemente se deban a la disponibilidad de los elementos en el suelo en cada sitio con sus condiciones ambientales, así como a diferencias en factores climáticos que pudieron afectar su absorción.

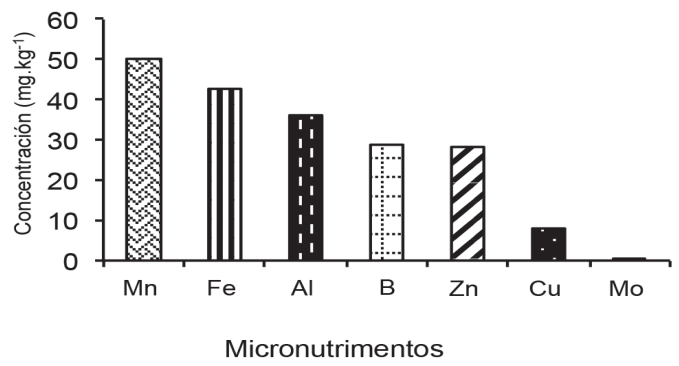

Fig. 9. Concentración de macro y microelementos foliares en teca de 3 a 8 años en la Cuenca del Canal Panamá. 
El ámbito de valores de concentración del N, Ca y S foliar determinado para Panamá está dentro de la variación reportada para África, aunque la amplitud de valores para Panamá es un poco menor (Cuadro 6). Los macroelementos $\mathrm{P}$ y K (Cuadro 6) y todos los microelementos (Cuadro 7), excepto el B y el Zn, se encuentran en concentraciones menores que las registradas en África.

Cuadro 6. Valores de concentración foliar (\%) de macronutrimentos en plantaciones de teca.

\begin{tabular}{lccccccc}
\hline \multicolumn{1}{c}{ País } & $\mathrm{N}$ & $\mathrm{P}$ & $\mathrm{K}$ & $\mathrm{Ca}$ & $\mathrm{Mg}$ & & Autor \\
& & & $\%$ & & & Orden mayor a menor & \\
\hline Panamá & 1,90 & 0,12 & 0,66 & 1,06 & 0,27 & $\mathrm{~N}>\mathrm{Ca}>\mathrm{K}>\mathrm{Mg}>\mathrm{P}$ & Presente trabajo 2012 \\
Costa Rica & 2,01 & 0,2 & 1,40 & 0,97 & 0,28 & $\mathrm{~N}>\mathrm{K}>\mathrm{Ca}>\mathrm{Mg}>\mathrm{P}$ & Montero y Kanninen (2006) \\
África & 2,15 & 0,20 & 1,56 & 1,46 & 0,29 & $\mathrm{~N}>\mathrm{K}>\mathrm{Ca}>\mathrm{Mg}>\mathrm{P}$ & Dreschel y Zech (1991) \\
India & 1,14 & 0,05 & 0,67 & 1,18 & 0,27 & $\mathrm{Ca}>\mathrm{N}>\mathrm{K}>\mathrm{Mg}>\mathrm{P}$ & Negi et ál (1990) \\
Nigeria & 1,41 & 0,29 & 2,57 & 0,90 & 0,19 & $\mathrm{~K}>\mathrm{N}>\mathrm{Ca}>\mathrm{P}>\mathrm{Mg}$ & Nwoboshi (1983, 1984) \\
\hline
\end{tabular}

Cuadro 7. Valores de concentración foliar $\left(\mathrm{mg} \cdot \mathrm{kg}^{-1}\right)$ de microelementos en plantaciones de teca.

\begin{tabular}{lcccccccc}
\hline \multicolumn{1}{c}{ País } & Fe & $\mathrm{Cu}$ & $\mathrm{Mn}$ & $\mathrm{B}$ & $\mathrm{Zn}$ & $\mathrm{Al}$ & Orden & Autor \\
\hline Panamá & 43 & 8 & 50 & 29 & 28 & 36 & $\mathrm{Mn}>\mathrm{Fe}>\mathrm{Al}>\mathrm{B}>\mathrm{Zn}>\mathrm{Cu}$ & Presente trabajo 2012 \\
África & 224 & 18 & 81 & 30 & 35 & 202 & $\mathrm{Fe}>\mathrm{Al}>\mathrm{Mn}>\mathrm{B}>\mathrm{Zn}>\mathrm{Cu}$ & Dreschel y Zech (1991) \\
Costa Rica & $\mathrm{Nd}$ & 14 & 49 & $\mathrm{Nd}$ & 30 & $\mathrm{nd}$ & $\mathrm{Mn}>\mathrm{Zn}>\mathrm{Cu}$ & $\begin{array}{l}\text { Montero y Kanninen } \\
(2006)\end{array}$ \\
\hline
\end{tabular}

Los valores de los nutrimentos $\mathrm{N}, \mathrm{P}, \mathrm{K}, \mathrm{Ca}$, $\mathrm{S}, \mathrm{Fe}, \mathrm{Cu}, \mathrm{Al}, \mathrm{B}$ y el Mo muestran una distribución normal mientras que el $\mathrm{Mg}$, el Mn y el $\mathrm{Zn}$ exhiben un cierto sesgo a la derecha.

En el Cuadro 8 se muestran los valores de los intervalos de concentración foliar considerados como bajos, adecuados y altos para las plantaciones de teca en la zona del Canal de Panamá. Con base en los datos se determinó que el $80 \%$ de la distribución normal correspondía al límite adecuado de concentración foliar de cada uno de los nutrimentos. El ámbito adecuado de la concentración foliar de Ca en la teca de la cuenca del canal de Panamá es desde $0,72 \%$ hasta $1,42 \%$. 
Cuadro 8. Intervalos (bajo, adecuado y alto) de concentración foliar para los macro y microelementos en teca de 3 a 8 años en Panamá.

\begin{tabular}{|c|c|c|c|c|}
\hline Variable & & Bajo & Adecuado & Alto \\
\hline \multirow{6}{*}{ Macro-elementos (\%) } & $\mathrm{N}$ & $<1,58$ & $>=1,58 \mathrm{y}<=2,19$ & $>2,19$ \\
\hline & $\mathrm{P}$ & $<0,10$ & $>=0,10 \mathrm{y}<=0,14$ & $>0,14$ \\
\hline & K & $<0,45$ & $>=0,45 \mathrm{y}<=0,89$ & $>0,89$ \\
\hline & $\mathrm{Ca}$ & $<0,72$ & $>=0,72 \mathrm{y}<=1,42$ & $>1,42$ \\
\hline & $\mathrm{Mg}$ & $<0,16$ & $>=0,16 \mathrm{y}<=0,44$ & $>0,44$ \\
\hline & S & $<0,17$ & $>=0,17 \mathrm{y}<=0,23$ & $>0,23$ \\
\hline \multirow{7}{*}{$\begin{array}{l}\text { Micro- elementos } \\
\quad\left(\mathrm{mg} \cdot \mathrm{kg}^{-1}\right)\end{array}$} & $\mathrm{Fe}$ & $<37$ & $>=37 \mathrm{y}<=50$ & $>50$ \\
\hline & B & $<23$ & $>=23 \mathrm{y}<=36$ & $>36$ \\
\hline & $\mathrm{Cu}$ & $<6$ & $>=6 \mathrm{y}<=11$ & $>11$ \\
\hline & $\mathrm{Al}$ & $<28$ & $>=28 \mathrm{y}<=46$ & $>46$ \\
\hline & Mo & $<0,25$ & $>=0,25 \mathrm{y}<=0,45$ & $>0,45$ \\
\hline & $\mathrm{Zn}$ & $<19$ & $>=19 \mathrm{y}<=39$ & $>39$ \\
\hline & $\mathrm{Mn}$ & $<25$ & $>=25 \mathrm{y}<=75$ & $>75$ \\
\hline
\end{tabular}

\section{CONCLUSIONES}

El orden registrado de la concentración foliar de macroelementos en teca en la cuenca del Canal de Panamá fue el N (1,9\%), Ca (1,06\%), K $(0,66 \%), \operatorname{Mg}(0,27 \%), \mathrm{S}(0,2 \%)$ y el P $(0,12 \%)$.

El orden de la concentración foliar de los microelementos en la teca en la cuenca del Canal de Panamá fue el Mn (50\%), Fe (43\%), Al (36\%), B (29\%), Zn (28\%) y el Cu (8\%) mg.kg-1.

El comportamiento de las curvas de concentración foliar de los macronutrimentos según la edad es variable: el $\mathrm{Ca}$ y el $\mathrm{Mg}$ aumentan con la edad, mientras que el $\mathrm{N}, \mathrm{P}$ y el $\mathrm{K}$ disminuyen conforme aumenta la edad.

La concentración de $\mathrm{N}$ y $\mathrm{P}$ foliar es explicada en un $49 \%$ y $43 \%$ respectivamente, por la edad de la teca entre 36 y 96 meses.

Los nutrimentos $\mathrm{P}, \mathrm{K}, \mathrm{Fe}$ y $\mathrm{Cu}$ se registraron en niveles marginales y deficientes en la cuenca del canal de Panamá en relación con los ámbitos definidos para teca en África (Dreschel y Zech 1991).

La concentración foliar de $\mathrm{Ca}$ es estadísticamente diferente en los 3 grupos de edad evaluados en este estudio (3-4 años / 7-8 años, $\mathrm{p}<0,001),(3-4$ años / 5-8 años, $\mathrm{p}<0,01)$.

La concentración foliar de $\mathrm{N}$ es estadísticamente diferente en los 3 grupos de edad evaluados en este estudio (3-4 años / 7-8 años, $\mathrm{p}<0,01$ ), (3-4 años / 5-8 años, p<0,05).

\section{LITERATUTA CITADA}

ALVARADO A., FALLAS J. 2004. La saturación de acidez y el encalado sobre el crecimiento de la teca (Tectona grandis L.f.) en suelos ácidos de Costa Rica. Agronomía Costarricense 28(1):81-87.

ÁVILA R.I. 2003. Evaluación del estado y crecimiento inicial de cuatro especies prioritarias (Pinus maximinoi H.E. Moore, Pinus caribaea, Pinus oocarpa schiede y Tectona grandis L.f.) del Programa de Incentivos Forestales en la región 2, en los departamentos de alta y Baja Verapaz, Guatemala. Tesis de maestría, CATIE, Turrialba, Costa Rica. 154 p.

BERTSCH F., CORRALES M., BEJARANO J. 2005. Los Laboratorios de análisis de suelos y foliares en Costa Rica: Informe del Comité de Laboratorios de análisis de suelos, plantas y aguas. Agronomía Costarricense 29(3):125-135.

BOARDMAN R., McGUIRE D.O. 1990. The role of zinc in forestry. I Zinc in forest environments, ecosystems 
and tree nutrition. Forest Ecology and Management 37:167-205.

DRECHSEL P., ZECH W. 1991. Foliar nutrient levels of broad-leaved tropical trees: A tabular review. Plant and Soil 131:29-46.

DRECHSEL P.,ZECH W. 1992. Multiple mineral deficiencies in forest plantations in Liberia. Forest Ecology and Management 48:121-143.

DRECHSEL P., ZECH W. 1994. DRIS evaluation of teak (Tectona grandis L.f.) mineral nutrition and effects of nutrition and site quality on teak growth in West Africa. Forest Ecology and Management 70:121-133.

HENRÍQUEZ C., BERTSCH F., SALAS R. 1995. Fertilidad de suelo: Manual de laboratorio. San José, CR, Asociación Costarricense de la Ciencia del Suelo. $64 \mathrm{p}$.

EVANS J. 1979. The effects of leaf position and leaf age in foliar analysis of Gmelina arborea. Plant and Soil 52:547-552.

FAO (ORGANIZACIÓN DE LAS NACIONES UNIDAS PARA LA AGRICULTURA Y LA ALIMENTACIÓN) 1977. Elaboración de una tabla de volumen y un estudio de incremento para teca (Tectona grandis) en El Salvador. Documento de trabajo $\mathrm{N}^{\circ}$. 14 FO:DP ELS/73/004. 53 p.

HERNÁNDEZ R., TORRES A., MÁRQUEZ O., FRANCO W. 1993. Contenido foliar y crecimiento en plantaciones de teca en Ticoporo, Venezuela. Turrialba 43(1):11-15.

KEOGH R.M. 1979. El futuro de la Teca en la América Tropical: Estudio sobre Tectona grandis en el Caribe, Centro América, Venezuela y Colombia. Roma. IT. FAO. Unasylva 31(126):13-19.

KOPPAD A., RAO R. 2003. Effect of moisture conservation methods and fertilizers on nutrient uptake in two-old teak (Tectona grandis L.f.) plantation, pp. 206-211. In: K. Bhat et ál. Proceedings of the International Conference on Quality Timber Products of Teak from Sustainable Forest Management. India.

LENOBLE M.E., BLEVINS D.G., SHARP R.E., CUMBIE B.G. 1996(a). Prevention of aluminum toxicity with supplemental boron. I. Maintenance of root elongation and celular structure. Plant, Cell and Enviromental 19:1132-1142.

LENOBLE M.E., BLEVINS D.G., LILES R.J. 1996(b). Prevention of aluminum toxicity with supplemental boron. II. Stimulation of root growth in an acidic, high-aluminun subsoil. Plant, Cell and Enviromental 19:1143-1148

MOLLINEDO M., UGALDE L., ALVARADO A., VERJANS J.M., RUDY L.C. 2005. Relación sueloárbol y factores de sitio, en plantaciones jóvenes de teca (Tectona grandis L.f.), en la zona oeste de la cuenca del Canal de Panamá. Agronomía Costarricense 29(1):67-75.
MONTERO M. 1999. Factores de sitio que influyen en el crecimiento de Tectona grandis L.f. y Bombacopsis quinatum (Jacq). Dugand en Costa Rica. Tesis de maestría, Universidad Austral de Chile, Valdivia, Chile. 111 p.

MONTERO M., KANNINEN M. 2006. Variación de los nutrimentos foliares con la edad de los árboles de Tectona grandis L.f., en Costa Rica. Proyecto: Dinámica de plantaciones. Universidad de Helsinki/ CATIE. Turrialba, Costa Rica. Sin publicar. 16 p.

NEGI J.D.S., BAHUGUNA V.K., SHARMA D.C. 1990. Biomass production and distribution of nutrients in 20 years old teak (Tectona grandis L.f.) and Gamar (Gmelina arborea) plantation in Tripura. The Indian Forester 116(9):681-686.

NWOBOSHI L. 1983. Growth and nutrient requirements in a teak plantation age series in Nigeria: I Linear growth and biomass production. Forest Science 29(1):159-165.

NWOBOSHI L. 1984. Growth and nutrient requirements in a teak plantation age series in Nigeria: II Nutrient accumulation and minimum annual requirements. Forest Science 30(1):35-40.

RODAS F. 2006. Efecto del establecimiento de plantaciones de teca (Tectona grandis L.f.) en áreas de potrero sobre las características del suelo en Petén, Guatemala. Tesis de maestria, CATIE, Turrialba, Costa Rica. 90 p.

SAMPAIO A., HUBER D. 1999. Concentração e distribuição de nutrimentos nas folhas de especies florestais, na Amazonia Ocidental. Floresta e Ambiente 6(1):127-137.

STATSOFT INC. 2001. STATISTICA (Data Analysis Software System), versión 6.

SUJATHA M.P. 2003. Significance of micronutrients on the growth of teak seedlings, pp. 491-494. In: K. Bhat et ál. Proceedings of the International Conference on Quality Timber products of Teak from Sustainable Forest Management. India.

TLBG (THE LOUIS BERGER GROUP INC. P). 2006. Informe de la región occidental de la cuenca del Canal de Panamá. Presentado a la Autoridad del Canal de Panamá. 21 p.

VAIDES E.E. 2004. Características de sitio que determinan el crecimiento y productividad de teca (Tectona grandis L.f.), en plantaciones forestales de diferentes regiones en Guatemala. Tesis de maestría, CATIE, Turrialba, Costa Rica. 95 p.

VALLEJOS O.S. 1996. Productividad y relaciones del índice de sitio con variables fisiográficas, edafoclimáticas y foliares para Tectona grandis L.f., Bombacopsis quinatum (Jacq) Dugand y Gmelina arbórea Roxb. en Costa Rica. Tesis de maestría, CATIE, Turrialba, Costa Rica. 147 p.

VÁSQUEZ W., UGALDE L.A. 1995. Rendimiento y calidad de sitio para Gmelina arborea, Tectona grandis, Bombacopsis quinata y Pinus caribaea, 
en Guanacaste, Costa Rica. CATIE/ IDA/ FAO/ HOLANDA. Proyecto Madelaña-3. Turrialba, Costa Rica. Serie Técnica, Informe Técnico, No. 256. 33 p. VIMAL M., SUDHAKARA K., IAYARAMAN K., SUNANDA C. 2003. Effect of soil-leaf nutritional factors of the productivity of teak (Tectona grandis L.f.) in Kerala State, India, pp. 530-539. In: Proceedings of the International Conference on Quality Timber Products of Teak from Sustainable Forest Management. Peechi, India. 2-5 December. 2003.
VITORELLO V.A., CAPALDI F.R., STEFANUTO V.A. 2005. Recent advances in aluminum toxicity and resistance in higher plants. Brazilian Journal of Plant Physiology 17(1):129-143.

ZECH W., KAUPENJOHANN M. 1990. Carences en potassium et en phosphore chez Casuarina equisetifolia, Eucalyptus sp. Acacia auriculiformis et Tectona grandis L.f. au SudBenin (Afrique occidentale). Bois et Forests des Tropiques 226:29-36. 